\title{
ORIENTATION CONTROL VIA A NON-MINIMAL STATE REPRESENTATION: THE PENDULUM CASE STUDY ${ }^{1}$
}

\author{
Ricardo Campa and Rafael Kelly \\ Dirección de Telemática, CICESE \\ Apdo. Postal 2615, Adm. 1, \\ Carretera Tijuana-Ensenada Km. 107 \\ Ensenada, B.C., 22800, MEXICO \\ e-mail: rkelly@cicese.mx,Fax: +52(646) 175-05-54
}

\begin{abstract}
Three degrees of freedom are required to completely describe the orientation (attitude) of a rigid body. Nevertheless, it is possible to use a non-minimal set of state variables, plus some holonomic constraints, for representing orientation (e.g. Euler parameters). This paper deals with orientation control problem using this latter approach, when the motion is constrained to a plane. The simplest testbed for such a motion is the pendulum. First, an alternative dynamic model of the pendulum is presented, which uses a non-minimal state representation. Once the orientation control objective for the plane is established, two controllers that solve this problem are introduced, and LaSalle's invariance principle is used to show the achievement of the control aim. Copyright (c) 2005 IFAC
\end{abstract}

Keywords: Orientation control, Non-minimal realization, Invariance principle, Euler parameters, Pendulum.

\section{INTRODUCTION}

The number of degrees of freedom required to completely define the pose of a rigid body is six, being three for position and three for orientation. According to this, a minimal set of three independent variables should be used for any representation of the orientation. Traditionally, three Euler angles are employed. But, in spite of their popularity, Euler angles suffer from representation singularities.

To avoid this drawback, and also solve the problem of orientation control, in the recent years some other non-minimal parameterizations of orienta-

\footnotetext{
1 Work partially supported by CONACyT, and CYTED.
}

tion have been studied (see e.g., Spring (1986), Wen and Kreutz-Delgado (1991)). Examples are the rotation matrices, the angle-axis pair, and the Euler parameters. All of them use a set of $3+k$ parameters, related by $k$ holonomic constraints, in order to keep the required three degrees of freedom.

Of particular interest are the Euler parameters. They are four parameters with a unit norm constraint, so that they can be considered as unit quaternions that evolve on $\mathrm{S}^{3}$, the hypersphere of dimension three.

The main concern of this paper is to clarify the basic concepts related to the use of Euler parameters as a non-minimal parameterization of orientation. In order to do so, and also show the application of 
some controllers that use this approach to a simple mechanism, we have constrained the motion of the rigid body to only two dimensions (e.g. a plane). In this case, only three degrees of freedom are required (one for orientation), and the pendulum shows up as a useful system to model and control.

\section{ORIENTATION CONTROL PROBLEM}

The dynamics of a serial-chain $n$-link robot manipulator can be written in joint space as (Spong and Vidyasagar, 1989):

$$
M(\boldsymbol{q}) \ddot{\boldsymbol{q}}+C(\boldsymbol{q}, \dot{\boldsymbol{q}}) \dot{\boldsymbol{q}}+\boldsymbol{g}(\boldsymbol{q})=\boldsymbol{\tau}
$$

where $\boldsymbol{q}$ is the $n \times 1$ vector of joint displacements, $\dot{\boldsymbol{q}}$ is the $n \times 1$ vector of joint velocities, $\boldsymbol{\tau}$ is the $n \times 1$ vector of applied torque inputs, $M(\boldsymbol{q})$ is the $n \times n$ symmetric positive definite manipulator inertia matrix, $C(\boldsymbol{q}, \dot{\boldsymbol{q}}) \dot{\boldsymbol{q}}$ is the $n \times 1$ vector of centripetal and Coriolis torques and $\boldsymbol{g}(\boldsymbol{q})$ is the $n \times 1$ vector of gravitational torques.

In the general case, it is common to consider that the manipulator output $\boldsymbol{x}(\boldsymbol{q})$ is the pose (position and orientation) of a frame attached to the endeffector referred to a fixed base frame. But in this paper, for the sake of simplicity, let us consider that only the orientation is of concern, i.e. $\boldsymbol{x}$ denotes the end-effector orientation.

Euler parameters $\left[\begin{array}{ll}\eta & \varepsilon^{T}\end{array}\right]^{T} \in \mathrm{S}^{3} \subset \mathbb{R}^{4}$ are usually defined in terms of an angle $\theta \in \mathbb{R}$ and a unit vector $\boldsymbol{u} \in \mathrm{S}^{2}$, corresponding to the angleaxis representation of a rotation, due to Euler (Sciavicco and Siciliano, 2000):

$$
\boldsymbol{x}=\left[\begin{array}{l}
\eta \\
\varepsilon
\end{array}\right]=\left[\begin{array}{c}
\cos \left(\frac{\theta}{2}\right) \\
\sin \left(\frac{\theta}{2}\right) \boldsymbol{u}
\end{array}\right]
$$

where all, $\theta, \boldsymbol{u}, \eta$, and $\varepsilon$ can be obtained as a function of $\boldsymbol{q}$. The angular velocity of the endeffector, denoted by $\boldsymbol{\omega} \in \mathbb{R}^{3}$, is obtained via the differential kinematics by:

$$
\boldsymbol{\omega}=J(\boldsymbol{q}) \dot{\boldsymbol{q}}
$$

where $J(\boldsymbol{q}) \in \mathbb{R}^{n \times n}$ is the manipulator geometric Jacobian. As pointed out by Campa et al. (2001), $\eta, \varepsilon$, and $\boldsymbol{\omega}$ can also be obtained directly from the corresponding rotation matrix, $R \in \mathrm{SO}(3)$.

Observe that the Euler parameters $\eta$ and $\varepsilon$ satisfy the next holonomic constraint

$$
\eta^{2}+\|\varepsilon\|^{2}=1
$$

Also, it can be shown that $\left[\begin{array}{ll}\eta & \varepsilon\end{array}\right]^{T}$ and $\left[\begin{array}{ll}-\eta & -\varepsilon\end{array}\right]^{T}$ represent the same orientation.

The time derivative of the Euler parameters is established by the so-called quaternion propagation rule (Sciavicco and Siciliano, 2000):

$$
\begin{aligned}
& \dot{\eta}=-\frac{1}{2} \varepsilon^{T} \boldsymbol{\omega} \\
& \dot{\varepsilon}=\frac{1}{2}[\eta I-S(\varepsilon)] \boldsymbol{\omega} .
\end{aligned}
$$

where, for a given $\boldsymbol{x} \in \mathbb{R}^{3}$, the skew-symmetric matrix $S(\boldsymbol{x})$ is defined by

$$
S(\boldsymbol{x})=\left[\begin{array}{ccc}
0 & -x_{3} & x_{2} \\
x_{3} & 0 & -x_{1} \\
-x_{2} & x_{1} & 0
\end{array}\right]
$$

\subsection{Orientation error and control objective}

The desired orientation trajectory is specified by the desired Euler parameters $\eta_{d}(t)$ and $\varepsilon_{d}(t)$, the desired angular velocity $\boldsymbol{\omega}_{d}(t)$ and the desired angular acceleration $\dot{\boldsymbol{\omega}}_{d}(t)$.

The angular velocity error vector $\widetilde{\boldsymbol{\omega}} \in \mathbb{R}^{3}$ is defined by

$$
\widetilde{\boldsymbol{\omega}}=\boldsymbol{\omega}_{d}-\boldsymbol{\omega}
$$

and, for the orientation error, let us consider

$$
\begin{aligned}
& \widetilde{\eta}=\eta \eta_{d}+\varepsilon^{T} \varepsilon_{d} \\
& \widetilde{\varepsilon}=\eta \varepsilon_{d}-\eta_{d} \varepsilon+S(\varepsilon) \varepsilon_{d}
\end{aligned}
$$

It can be shown that the pair $(\widetilde{\eta}, \widetilde{\varepsilon})$ forms a unit quaternion (i.e. $\left[\begin{array}{ll}\widetilde{\eta} & \widetilde{\varepsilon}^{T}\end{array}\right]^{T} \in \mathrm{S}^{3}$ ) and it corresponds to the Euler parameters for the mutual orientation between $\left[\begin{array}{ll}\eta_{d} & \varepsilon_{d}^{T}\end{array}\right]^{T}$ and $\left[\begin{array}{ll}\eta & \varepsilon^{T}\end{array}\right]^{T}$. Such a definition of the orientation error was first used by Yuan (1988), and then by other authors (Lin, 1995; Caccavale et al., 1999; Sciavicco and Siciliano, 2000).

The orientation control objective is to achieve that the orientation of the manipulator's endeffector follows the desired orientation. For this, we can use any representation of the orientation. In the case of using Euler parameters the control objective is

$$
\lim _{t \rightarrow \infty}\left[\begin{array}{l}
\eta(t) \\
\varepsilon(t)
\end{array}\right]=\left[\begin{array}{l}
\eta_{d}(t) \\
\varepsilon_{d}(t)
\end{array}\right],
$$

or, what is the same

$$
\lim _{t \rightarrow \infty}\left[\begin{array}{c}
|\widetilde{\eta}(t)| \\
\widetilde{\varepsilon}(t)
\end{array}\right]=\left[\begin{array}{l}
1 \\
\mathbf{0}
\end{array}\right] .
$$

Yuan (1988) showed that this control objective is satisfied if and only if $\lim _{t \rightarrow \infty} \widetilde{\varepsilon}(t)=\mathbf{0}$.

We digress momentarily to establish an application of the LaSalle's invariance principle which is fundamental to the purpose of this paper. 
Theorem 1. Consider the autonomous system

$$
\dot{\boldsymbol{x}}=\boldsymbol{f}(\boldsymbol{x})
$$

where $\boldsymbol{f}: D \rightarrow \mathbb{R}^{n}$ is a Lipschitz map from a domain $D \subset \mathbb{R}^{n}$ into $\mathbb{R}^{n}$. Let $V: D \rightarrow \mathbb{R}$ be a continuous differentiable function such that

$$
V(\boldsymbol{x}) \rightarrow \infty \text { as }\|\boldsymbol{x}\| \rightarrow \infty,
$$

and

$$
\dot{V}(\boldsymbol{x}) \leq 0 \text { for all } \boldsymbol{x} \in D
$$

Let $\Omega$ be

$$
\Omega=\{\boldsymbol{x} \in D: \dot{V}(\boldsymbol{x})=0\}
$$

and $M$ be the largest invariant set in $\Omega$. Then

$$
\lim _{t \rightarrow \infty} \operatorname{dist}(\boldsymbol{x}(t), M)=0 \quad \forall \boldsymbol{x}(0) \in D .
$$

Proof. The proof relies on application of Theorem 4.4 from Khalil (2001). To this end, define the closed set $D_{c} \subset D$ as

$$
D_{c}=\{\boldsymbol{x} \in D: V(\boldsymbol{x}) \leq c\}
$$

for $c>0$. In virtue of the radially unboundedness assumption on function $V(\boldsymbol{x})$ in (14), the boundedness of set $D_{c}$ is guaranteed for all values of $c$. This fact and (15) imply that $D_{c}$ is also a positively invariant set with respect to (13) for all values of $c$. Both features of $D_{c}$-being a compact and positively invariant set- are needed for application of Theorem 4.4 in Khalil (2001).

On the other hand, define the set $\Omega_{c} \subset \Omega$ as

$$
\Omega_{c}=\left\{\boldsymbol{x} \in D_{c}: \dot{V}(\boldsymbol{x})=0\right\} .
$$

Hence, from (15) results

$$
\dot{V}(\boldsymbol{x}) \leq 0 \text { for all } \boldsymbol{x} \in D_{c}
$$

which is valid for all values of $c$. Then, invoking Theorem 4.4 in Khalil (2001) we have

$$
\lim _{t \rightarrow \infty} \operatorname{dist}\left(\boldsymbol{x}(t), M_{c}\right)=0 \quad \forall \boldsymbol{x}(0) \in D_{c}
$$

where $M_{c}$ is the largest invariant set in $\Omega_{c}$. Since $c$ can be arbitrarily large, then we have the conclusion (17).

\section{A CASE STUDY: THE PENDULUM}

The pendulum is a simple and intuitive nonlinear physical system. Figure 1 shows a diagram of a pendulum with its distinctive parameters.

For the physical description of the pendulum, let us consider the following parameters: $m$, the total
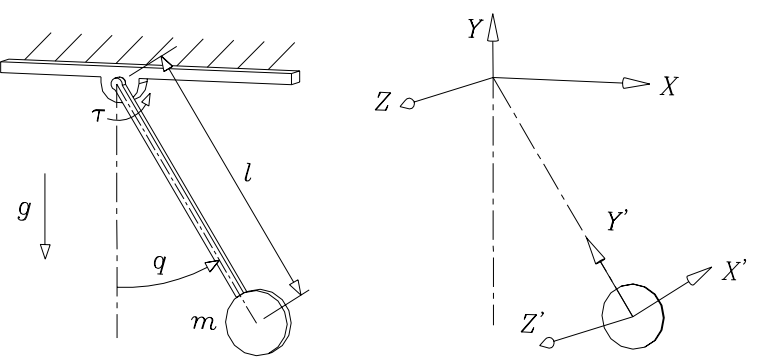

Fig. 1. Simple pendulum

mass of the pendulum, concentrated in the center of mass; $l$, the distance between the axis of rotation and the center of mass; $\tau$, the external torque applied to the pendulum axis; $g$, the acceleration due to gravity. The differential equation that rules the motion of the pendulum is:

$\frac{d}{d t}\left[\begin{array}{c}q \\ \dot{q}\end{array}\right]=\left[\begin{array}{c}\dot{q} \\ \frac{1}{m l^{2}}(\tau-m g l \sin (q))\end{array}\right],\left[\begin{array}{c}q \\ \dot{q}\end{array}\right] \in \mathbb{R}^{2}$

where $q$ is the angle of the pendulum respect to the vertical and $\dot{q}$ is the corresponding angular velocity.

\subsection{Non-minimal realization}

System (18) uses the minimum number of variables (states) required to describe completely the motion of the pendulum. However, it is also possible to use a non-minimal realization.

Let us consider the next definition of variables:

$$
\eta=\cos \left(\frac{q}{2}\right) ; \varepsilon=\sin \left(\frac{q}{2}\right)
$$

so that the holonomic constraint

$$
\eta^{2}+\varepsilon^{2}=1
$$

is always satisfied. So, we have that $\left[\begin{array}{ll}\eta & \varepsilon\end{array}\right]^{T} \in \mathrm{S}$, i.e. it evolves on the unit circle.

Figure 2 shows how $\eta$ and $\varepsilon$ can be interpreted physically in the pendulum. Notice that dividing by 2 the angle $q$ causes the period of the trigonometric functions to double. If, for a given angle $q$ we have a pair $\left[\begin{array}{ll}\eta & \varepsilon\end{array}\right]^{T}$, then for $q+2 \pi$ (which topologically corresponds to the same angle) we have $\left[\begin{array}{ll}-\eta & -\varepsilon\end{array}\right]^{T}$.

It is clear that parameters $\eta$ and $\varepsilon$ are related to the Euler parameters described in Section 2. In fact, they are a particular case, when the motion is restricted to the plane, and only one degree of freedom is required for orientation. To see this, consider the following for the case of the pendulum.

Suppose that the plane of the motion is the $X-Y$ plane in the 3D Cartesian space. Then, the only possible rotation must be accomplished along the 

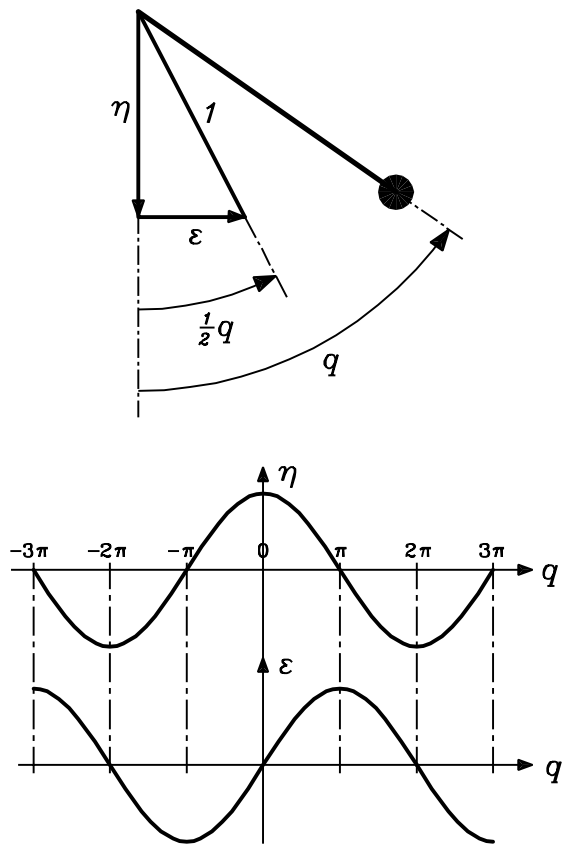

Fig. 2. Physical interpretation of $\eta$ and $\varepsilon$

axis $\boldsymbol{u}=\left[\begin{array}{lll}0 & 0 & 1\end{array}\right]^{T}$. The only variable describing orientation is the angle $q$ which corresponds to the $\theta$ variable of the angle-axis representation. Then, according to (2) the Euler parameters for the pendulum are $\eta=\cos \left(\frac{q}{2}\right)$ and $\varepsilon=\left[\begin{array}{lll}0 & 0 & \sin \left(\frac{q}{2}\right)\end{array}\right]^{T}$. Also, notice that $\boldsymbol{\omega}=\left[\begin{array}{lll}0 & 0 & \omega\end{array}\right]^{T}$, where $\omega=\dot{\theta}=$ $\dot{q}$.

So, the four-parameter description of orientation given by the Euler parameters reduces to only two non-zero parameters in the case of twodimensional motion. These two parameters correspond precisely to the new variables defined in (19) and their physical significance has been explained above.

To obtain the time derivatives of $\eta$ and $\varepsilon$ for the pendulum, we can simply derivate from (19) or substitute the corresponding terms in the quaternion propagation rule (5)-(6). We obtain:

$$
\dot{\eta}=-\frac{1}{2} \varepsilon \omega, \quad \dot{\varepsilon}=\frac{1}{2} \eta \omega .
$$

On the other hand, we can use some trigonometric identities to get the following useful relations:

$$
\cos (q)=\eta^{2}-\varepsilon^{2} \quad ; \quad \sin (q)=2 \eta \varepsilon
$$

Taking $\eta, \varepsilon$, and $\omega$, as the new state variables, the pendulum dynamics (18) is expressed as:

$$
\frac{d}{d t}\left[\begin{array}{l}
\eta \\
\varepsilon \\
\omega
\end{array}\right]=\left[\begin{array}{c}
-\frac{1}{2} \varepsilon \omega \\
\frac{1}{2} \eta \omega \\
\frac{1}{M}[\tau-2 m g l \eta \varepsilon]
\end{array}\right]
$$

where $M=m l^{2}$ with $\left[\begin{array}{l}\eta \\ \varepsilon \\ \omega\end{array}\right] \in \mathrm{S} \times \mathbb{R} \subset \mathbb{R}^{3}$.

\section{TWO ORIENTATION CONTROLLERS}

This section introduces two orientation controllers for the pendulum, which make use of a nonminimal representation for the orientation. In the case of motion in the plane, the orientation error reduces to (see (9)-(10)):

$$
\begin{aligned}
& \widetilde{\eta}=\eta \eta_{d}+\varepsilon \varepsilon_{d} \\
& \widetilde{\varepsilon}=\eta \varepsilon_{d}-\eta_{d} \varepsilon .
\end{aligned}
$$

where $\left[\begin{array}{ll}\eta_{d} & \varepsilon_{d}\end{array}\right]^{T} \in \mathrm{S}$ corresponds to the desired orientation, and can be obtained from a desired angle $q_{d}$ through a map similar to (19). Also, notice that $\left[\begin{array}{cc}\widetilde{\eta} & \widetilde{\varepsilon}\end{array}\right]^{T} \in \mathrm{S}$ is obtained applying the same map to the angle $\widetilde{q}=q_{d}-q$. Moreover, the angular velocity error is defined as $\widetilde{\omega}=\omega_{d}-\omega$, where $\omega_{d}$ is the desired angular velocity.

By using (20), and the fact that $\eta_{d}^{2}+\varepsilon_{d}^{2}=1$, it can be shown that $\left[\begin{array}{ll}\widetilde{\eta} & \widetilde{\varepsilon}\end{array}\right]$, as defined in (23)-(24), also satisfies the unit norm constraint, i.e.

$$
\widetilde{\eta}^{2}+\widetilde{\varepsilon}^{2}=1
$$

The orientation control objective in the plane is ensured if

$$
\lim _{t \rightarrow \infty} \widetilde{\varepsilon}(t)=0
$$

For the analysis below, it is convenient to define the domain $D \subset \mathbb{R}^{3}$ as:

$D=\mathrm{S} \times \mathbb{R}=\left\{\left[\begin{array}{c}\widetilde{\eta} \\ \widetilde{\varepsilon} \\ \widetilde{\omega}\end{array}\right] \in \mathbb{R}^{3}: \widetilde{\eta}^{2}+\widetilde{\varepsilon}^{2}-1=0\right\}$.

\subsection{Resolved acceleration control}

First proposed by Luh et al. (1980) for solving the general problem of task space control. In the case of the pendulum, this controller reduces to:

$$
\tau=M\left[\dot{\omega}_{d}+k_{v} \widetilde{\omega}+k_{p} \widetilde{\varepsilon}\right]+2 m g l \eta \varepsilon
$$

where $k_{p}$ and $k_{v}$ are strictly positive control gains.

By substituting the control law (28) into pendulum dynamics (22) we get the closed-loop equation:

$$
\dot{\widetilde{\omega}}+k_{v} \widetilde{\omega}+k_{p} \widetilde{\varepsilon}=0
$$

which can be expressed in terms of the state vector $\left[\begin{array}{lll}\widetilde{\eta} & \widetilde{\varepsilon} & \widetilde{\omega}\end{array}\right]^{T}$ as:

$$
\frac{d}{d t}\left[\begin{array}{c}
\widetilde{\eta} \\
\widetilde{\varepsilon} \\
\widetilde{\omega}
\end{array}\right]=\left[\begin{array}{c}
-\frac{1}{2} \widetilde{\varepsilon \omega} \\
\frac{1}{2} \widetilde{\eta} \widetilde{\omega} \\
-k_{p} \widetilde{\widetilde{\varepsilon}}-k_{v} \widetilde{\omega}
\end{array}\right],\left[\begin{array}{l}
\widetilde{\eta} \\
\widetilde{\varepsilon} \\
\widetilde{\omega}
\end{array}\right] \in D .
$$

This is an autonomous system, with equilibria in the set (see Figure 3):

$\mathcal{S}=\left\{\left[\begin{array}{lll}\widetilde{\eta} & \widetilde{\varepsilon} & \widetilde{\omega}\end{array}\right]^{T} \in D:|\widetilde{\eta}|=1, \widetilde{\varepsilon}=0, \widetilde{\omega}=0\right\}$. 


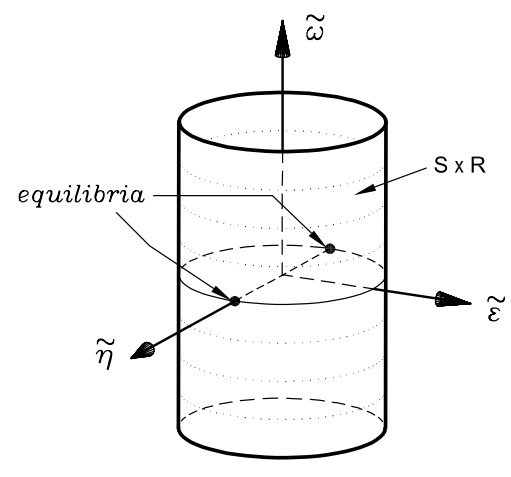

Fig. 3. Domain of definition of the closed-loop system

Notice that $\mathcal{S}$ has only two elements, that is, the equilibria are at

$$
\left[\begin{array}{c}
\widetilde{\eta} \\
\widetilde{\varepsilon} \\
\widetilde{\omega}
\end{array}\right]=\left[\begin{array}{l}
1 \\
0 \\
0
\end{array}\right] \quad \text { and } \quad\left[\begin{array}{c}
\widetilde{\eta} \\
\widetilde{\varepsilon} \\
\widetilde{\omega}
\end{array}\right]=\left[\begin{array}{c}
-1 \\
0 \\
0
\end{array}\right] .
$$

Notwithstanding, according to the definition of $\tilde{\eta}$ they correspond to the cases where $\widetilde{q}=0$ and $\widetilde{q}=2 \pi$, respectively, which represent the same orientation.

In order to analyze the system behavior, we shall utilize Theorem 1. To this end, consider the following function, which has been inspired from Lin (1995):

$$
V(\widetilde{\eta}, \widetilde{\varepsilon}, \widetilde{\omega})=[\widetilde{\eta}-1]^{2}+\widetilde{\varepsilon}^{2}+\frac{1}{2 k_{p}} \widetilde{\omega}^{2} .
$$

It is easy to verify that above function is radially unbounded in the sense (14). Obtaining the time derivative of (31), along the trajectories defined by (29) we get:

$$
\dot{V}(\widetilde{\eta}, \widetilde{\varepsilon}, \widetilde{\omega})=-\frac{k_{v}}{k_{p}} \widetilde{\omega}^{2} \leq 0 \quad \forall\left[\begin{array}{l}
\widetilde{\eta} \\
\widetilde{\varepsilon} \\
\widetilde{\omega}
\end{array}\right] \in D .
$$

On the other hand, the set $\Omega$ defined as (16) results in

$$
\Omega=\left\{\left[\begin{array}{lll}
\widetilde{\eta} & \widetilde{\varepsilon} & \widetilde{\omega}
\end{array}\right]^{T} \in D: \widetilde{\eta}^{2}+\widetilde{\varepsilon}^{2}-1=0, \widetilde{\omega}=0\right\} .
$$

It is easy to see that the largest invariant set in $\Omega$ is the equilibria set $\mathcal{S}$. Therefore, invoking Theorem 1 it can be concluded that the closedloop system trajectories tend asymptotically to the equilibria, i.e.

$\lim _{t \rightarrow \infty} \operatorname{dist}\left(\left[\begin{array}{l}\widetilde{\eta}(t) \\ \widetilde{\varepsilon}(t) \\ \widetilde{\omega}(t)\end{array}\right]-\mathcal{S}\right)=0 \quad \forall\left[\begin{array}{l}\widetilde{\eta}(0) \\ \widetilde{\varepsilon}(0) \\ \widetilde{\omega}(0)\end{array}\right] \in D$.

This ensures that the control objective (26) is achieved globally in the sense that any desired time-varying orientation $\left[\begin{array}{ll}\eta_{d}(t) & \varepsilon_{d}(t)\end{array}\right]^{T} \in \mathrm{S}$, is asymptotically tracked from any initial orientation.

\subsection{Slotine-Li type control}

This is an original controller, proposed to solve the problem of orientation control for the pendulum. It is motivated by the non-adaptive version of the controller introduced by Slotine and Li (1988). The proposed control law is given by:

$$
\tau=M\left[\dot{\omega}_{d}+\frac{1}{2} \lambda \widetilde{\eta} \widetilde{\omega}\right]+k_{p} \widetilde{\varepsilon}+k_{v} \widetilde{\omega}+2 m g l \eta \varepsilon
$$

where $k_{p} \mathrm{y} k_{v}$ are strictly positive control gains, and

$$
\lambda=k_{v}^{-1} k_{p}
$$

By substituting (32) into (22), we obtain the closed-loop equation

$$
M\left[\dot{\widetilde{\omega}}+\frac{1}{2} \lambda \widetilde{\eta} \widetilde{\omega}\right]+k_{p} \widetilde{\varepsilon}+k_{v} \widetilde{\omega}=0
$$

which can be also expressed in terms of the conventional states:

$\frac{d}{d t}\left[\begin{array}{c}\widetilde{\eta} \\ \widetilde{\varepsilon} \\ \widetilde{\omega}\end{array}\right]=\left[\begin{array}{c}-\frac{1}{2} \widetilde{\varepsilon} \widetilde{\omega} \\ \frac{1}{2} \widetilde{\eta} \widetilde{\omega} \\ \frac{1}{M}\left[-k_{p} \widetilde{\varepsilon}-k_{v} \widetilde{\omega}\right]-\frac{1}{2} \lambda \widetilde{\eta} \widetilde{\omega}\end{array}\right],\left[\begin{array}{c}\widetilde{\eta} \\ \widetilde{\varepsilon} \\ \widetilde{\omega}\end{array}\right] \in D$

where we can see that it is an autonomous system with equilibria in the set $\mathcal{S}$ defined in (30), that is, the same equilibria than for the resolved acceleration control system.

For the closed-loop analysis, we propose the following function inspired by the one introduced by Spong et al (1990):

$$
V(\widetilde{\eta}, \widetilde{\varepsilon}, \widetilde{\omega})=\frac{M}{2 k_{p}}[\widetilde{\omega}+\lambda \widetilde{\varepsilon}]^{2}+2 \widetilde{\varepsilon}^{2}+2[\widetilde{\eta}-1]^{2}
$$

which is radially unbounded in the sense of (14). The time derivative of (35) along the trajectories of system (34) yields:

$$
\dot{V}(\widetilde{\eta}, \widetilde{\varepsilon}, \widetilde{\omega})=-\lambda \widetilde{\varepsilon}^{2}-\lambda^{-1} \widetilde{\omega}^{2} .
$$

where we have used the definition of $\lambda$ (33), to simplify the terms. Notice that it satisfies

$$
\dot{V}(\widetilde{\eta}, \widetilde{\varepsilon}, \widetilde{\omega}) \leq 0 \quad \forall\left[\begin{array}{c}
\widetilde{\eta} \\
\widetilde{\varepsilon} \\
\widetilde{\omega}
\end{array}\right] \in D .
$$

Observe that the set $\Omega$ defined as (16) reduces to

$$
\Omega=\left\{\left[\begin{array}{lll}
\widetilde{\eta} & \widetilde{\varepsilon} & \widetilde{\omega}
\end{array}\right]^{T} \in D:|\widetilde{\eta}|=1, \widetilde{\varepsilon}=0, \widetilde{\omega}=0\right\} .
$$

This is trivially the invariant set because it corresponds to the equilibria set $\mathcal{S}$. Therefore, in virtue of the Theorem 1, the closed-loop system trajectories tend asymptotically to the equilibria, i.e.

$$
\lim _{t \rightarrow \infty} \operatorname{dist}\left(\left[\begin{array}{l}
\widetilde{\eta}(t) \\
\widetilde{\varepsilon}(t) \\
\widetilde{\omega}(t)
\end{array}\right]-\mathcal{S}\right)=0 \quad \forall\left[\begin{array}{c}
\widetilde{\eta}(0) \\
\widetilde{\varepsilon}(0) \\
\widetilde{\omega}(0)
\end{array}\right] \in D,
$$


hence, the control objective (26) is achieved globally in the sense that any desired time-varying orientation $\left[\begin{array}{ll}\eta_{d}(t) & \varepsilon_{d}(t)\end{array}\right]^{T} \in \mathrm{S}$, is tracked starting from any initial orientation.

\subsection{Discussion}

Simulations of the closed-loop systems (29) and (34) reveal that they have similar qualitative behavior. One equilibrium is asymptotically stable and the other, which is unstable, possesses a stable manifold. The flow of these systems is illustrated in Figure 4. As expected, the flow lines tend to the equilibria, so indicating the matching of the desired orientation (and desired angular velocity as well).
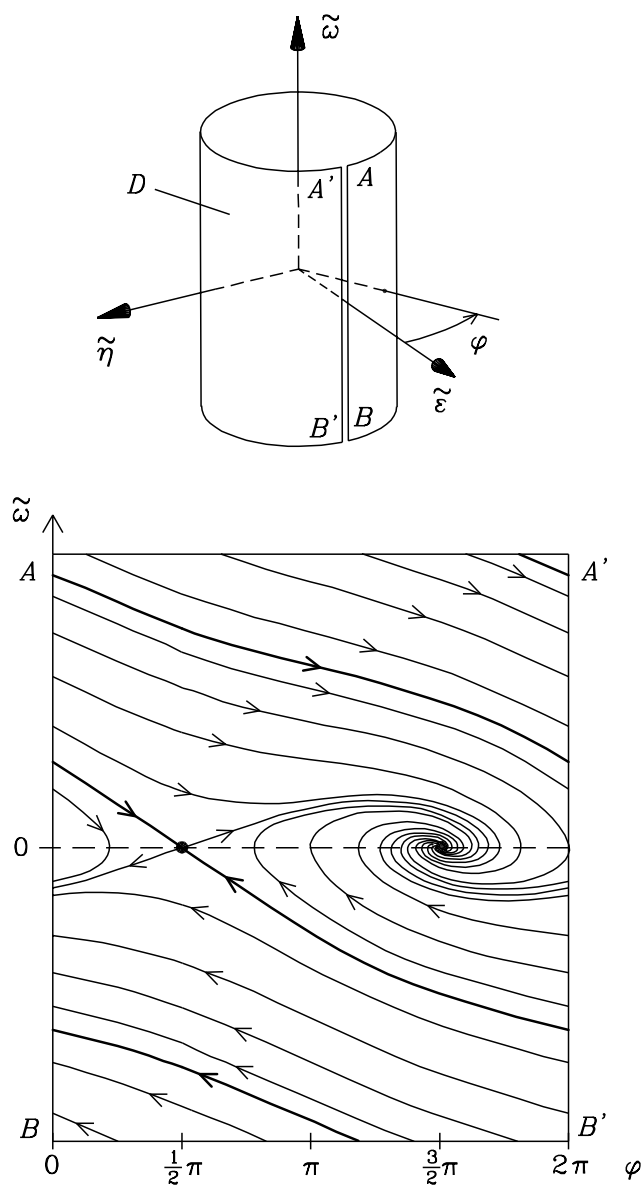

Fig. 4. Flow of the closed-loop systems as viewed on the plane

\section{CONCLUSION}

Although only three parameters are required to completely describe the orientation of a rigid body in space, there exist several representation for the orientation that use a non-minimal set of parameters. Among these representations, Euler parameters are commonly used because of their properties as unit quaternions. We have employed this approach to describe the pendulum dynamics, where the four Euler parameters reduce to only two.

In order to show the feasibility of this nonminimal state representation of the pendulum, we have studied two controllers that solve the orientation control problem in a plane. In both cases, we can conclude the fulfillment of the orientation tracking objective, by using an appropriate version of the LaSalle's invariance principle.

\section{REFERENCES}

Caccavale F., B. Siciliano and L. Villani (1999). The role of Euler parameters in robot control. Asian Journal of Control, Vol. 1, No. 1, pp. 25-34.

Campa R., R. Kelly and E. Garcia (2001). On stability of the resolved acceleration control. Proc. IEEE International Conference on Robotics and Automation, Vol. 4, pp. 3523-3528, Seoul, Korea.

Khalil H.K. (2001). Nonlinear systems, Prentice Hall, Second Edition.

Lin S. K. (1995). Robot control in Cartesian space. In: Progress in Robotics and Intelligent Systems (G.W. Zobrist and C.Y. Ho, Eds.). Vol. 3, pp. 85-124. Ablex Publishing, New Jersey.

Luh J.Y.S., M.W. Walker and R.P.C. Paul (1980). Resolved-acceleration Control of Mechanical Manipulators. IEEE Transactions on Automatic Control, Vol. 25, pp. 486-474.

Sciavicco L. and B. Siciliano (2000). Modeling and Control of Robot Manipulators, SpringerVerlag, London.

Slotine J. J. and W. Li (1988). Adaptive manipulator control: A case study, IEEE Trans. on Automatic Control, Vol. AC-33, No. 3, pp. 49-59.

Spong M.W. and M. Vidyasagar (1989). Robot Dynamics and Control, John Wiley and Sons, New York.

Spong M.W., R. Ortega and R. Kelly (1990). Comments on: Adaptive manipulator control: A case study, IEEE Trans. on Automatic Control, Vol. 35, No. 6, pp. 761-762.

Spring K.W. (1986). Euler parameters and the use of quaternion algebra in the manipulation of finite rotations: A review. Mechanism and Machine Theory, Vol. 21, No. 5, pp. 365-373.

Wen J.T.Y and K. Kreutz-Delgado (1991). The attitude control problem. IEEE Transactions on Automatic Control, Vol. 36, No. 10, pp. 1148-1162.

Yuan J.S.C. (1988) Closed-loop manipulator control using quaternion feedback. IEEE Journal of Robotics and Automation, Vol. 4, No. 4, pp. 434-440. 